\title{
Philosophiques
}

\section{Structure et causalité}

\section{J. N. Kaufmann}

Volume 3, numéro 1, avril 1976

URI : https://id.erudit.org/iderudit/203041ar

DOI : https://doi.org/10.7202/203041ar

Aller au sommaire du numéro

Éditeur(s)

Société de philosophie du Québec

ISSN

0316-2923 (imprimé)

1492-1391 (numérique)

Découvrir la revue

Citer cet article

Kaufmann, J. N. (1976). Structure et causalité. Philosophiques, 3(1), 3-32.

https://doi.org/10.7202/203041ar

Ce document est protégé par la loi sur le droit d'auteur. L'utilisation des services d'Érudit (y compris la reproduction) est assujettie à sa politique d'utilisation que vous pouvez consulter en ligne.

https://apropos.erudit.org/fr/usagers/politique-dutilisation/
Cet article est diffusé et préservé par Érudit.

Érudit est un consortium interuniversitaire sans but lucratif composé de l'Université de Montréal, l'Université Laval et l'Université du Québec à Montréal. Il a pour mission la promotion et la valorisation de la recherche. https://www.erudit.org/fr/ 


\section{STRUCTURE ET CAUSALité}

par J.N. Kaufmann

Depuis un demi-siècle, les logicien; des sciences sociales s'interrogent sur la légitimité et la portée de l'analyse causale dans les sciences de l'homme. Ce travail veut s'inscrire dans cette ligne. Les arguments qui y sont exposés soutiennent la thèse que l'analyse structurale est susceptible de remplacer l'analyse causale, à condition que le structuralisme réussisse à produire un type d'explication qui satisfasse les exigences de la « $H-D$ Explanation $\gg$ de Hempel.

Dans la premì̀re partie seront résumćes les diverses raisons des défenseurs et les multiples objections des adversaites de l'analyse causale. Ceci a pour but de montrer pourquoi l'usage du concept de «cause» est devenu problématique et est même tombí en discrédit. La deuxième partie contient un cas paradigmatique de l'analyse structurale. Il s'agit d'une reformulation de l'esquisse que fournit Lévi-Strauss pour une théorie générale de la parenté et pour un traitement structural de tous les phénomènes sociaux. On examinera dans une troisième partic le schéma structural de Lévi-Strauss par rapport à l'explication hempelienne, en vue d'établir s'il constitue une explication adćquate des problèmes (théoriques) et des faits qu'observent l'cthnologue et le sociologue, et si le schéma structural rejoint la réalité sociale dans la dimension qui rend l'analyse causale superflue.

\section{Lianalyse causale en sciences sociales}

La réticence de Newton à l'égard des «causes de la gravité », le discrédit que jette Hume sur la notion de «cause» comme concept empirique, son rejet radical par Wittgenstein dans le Tractatus et des raisons internes au développement des sciences ont contribué à éliminer le terme de «cause»du 
vocabulaire théorique de la physique contemporaine ${ }^{1}$. Il fallait par conséquent lui trouver un autre «lieu» que les sciences sociales allaient offrit. Mais son introduction a aussitôt soulevé des débats fort connus ${ }^{2}$ qui devaient néanmoins rester sans issue aussi longtemps qu'on ne distinguait pas clairement les questions relatives au statut des propositions calisales des questions ayant trait au principe de causclité. C'est finalement Durkheim qui accorde, au nom du détcrminisme sociologique qu'il défend dans les Règles et le Suicide, un rôle clef au concepto de «cause» dans l'analyse des faits sociaux, et c'est de hui, entre autres, que se réclameront les chercheurs contemporains".

Boudon est de l'avis qu'il est possible en sociologie d'assigner au concept de «cause» un sens empirique défini. Il maintiendrait aussi que les propositions causales sont vérifiables en principe, affirmation qui est discutable et effectivement discutée par plusieurs auteurs dont Simon qui soulèvera da nombrouses difficultés. Boudon va plus loin. Il prétend que le recours à lit causalité est indispensable. Il affirme qu'une corrélation statistique, la forme de relation de dépendance fonctionnelle la plus fréquente en sociologie, ne constitue pas, si elle n'est pas interprétée en termes de causalité, un énoncé empirique utile pour la sociologie, mais reste une proposition métathéorique ${ }^{4}$. De plus, les relations fonctionnelles étant parfaitement symútriques, elles ne sont d'aucune utilité dans des stratégies d'intervention puisqu'elles ne nous renseignent en rien sur le sens ou la direction de la dépendance des variables en jeu aussi longtemps qu'on ne

1. Cf. HEYDE, J.E., Entuertung der Katusalitat, Für und wider den Positivismus, Stuttgart (Kohlhammer), 1957. Il est à remarquer que «causalité », "cause » ou des termes semblables ne figurent pas dans l'index de Physics Abstracts. Mais Lélimination du concept sur le plan théorique ne présume en rien du rồie houristique du schéma causal dans un langage pré-scientifique, dans le contexte de lapplication d'une theorie ou dans colui des stratégics d'intervention.

2. Tandis que Rickert résiste à son usag» dans k's «Geisteswissenschaften » (RICKERT, H., Die Grenzen der natur-ulissenschaflichen Begriffsbilditnis, Tübingen (Mohr), 1913), Weber plaide en favcur de l'explication causale dans les sciences sociales (WEBER, M., Gesatimelte Aufsitze zur Wissenschaftslebre. Tübingen (Mohr), 1968, 3. Aufl.).

3. Voir BOUDON, R., L'enalyse mathimatique des faits sociatex, Paris, (Plon), 1967, ch. II ; ct BOUDON, R., LAZARSFELD, P., (cds.) L'analyse empirique de la causalite, Paris - LaHaye (Mouton \& Co.), 1966.

4. BOUDON, R., L'analyse matbématique des faits sociaux, op. cit., p. 22. 
donne pas une interprétation causale. En effet la question, à propos d'une corrélation statistique observée entre p.ex. «marginalité » et «apparition de symptômes dépressifs», est de savoir si la marginalité est la cause de l'apparition des symptômes ou l'inverse. La corrélation statistique comme telle ne l'indique pas. De là la nécessité, selon Boudon, d'une interprétation causale. (On soulèvera plus loin les difficultés inhérentes à une telle procédure.)

Boudon ne donne aucune définition du concept de «cause». Mais Simon, autre défenseur de l'analyse causale, en fournit une analyse ${ }^{5}$. La notion de «cause» appartiendrait à un métalangage à l'aide duquel on définit un ordre partiel sur les membres d'un ensemble de propositions atomiques, de sorte qu'une description d'état (proposition atomique) appartenant à un sous-ensemble qui est inclus dans un autre sous-ensemble a une priorité causale sur une description d'état appartenant au second sous-ensemble. Il est à remarquer que les sous-ensembles des propositions atomiques sont censés être des lois d'une théorie et on peut alors se poser la question si l'analyse causale en sociologie se réclame bien de lois qui vérifient la propriété formelle de s'inclure.

Simon est naturellement convaincu de l'importance et du rôle positif des propositions causales dans la recherche empirique. Mais il remarque qu'il est difficile d'assurer l'établissement d'un ordre causal zinique sur les éléments (descriptions d'états). L'unicité reposerait finalement sur la distinction entre des propositions qui sont de simples «observations » et des propositions qui sont tenues pour des «conditions», classification qui semble, même

5. Voir SIMON, H.A., Models of Man. Social and Rational. Mathematical Essays on Rational Human Behavior in a Social Setting, New York (John Wiley \& Sons, Inc.), 1957, notamment «On the Definition of the Causal Relation», pp. 50-62. Des analyses plus poussées peuvent ĉtre trouvées dans KIM, J., «Causation Nomic Subsumption and the Concept of Event », in: The Journal of Pbilosophy, vol. LXX, no 8, 1973, pp. 217.236 où on discute implicitement la définition que reprend Simon de Carnap et de Pap. Également chez MACKIE, J.L., «Causes and Conditions 》, in: American Pbilosophical Quarterley, vol. 2 no 4, 1965, pp. 245-264, texte qui examine principalement les propositions causales singulières où le concept de cause est analysé en termes de « inus-conditions 》 c'est-à-dire «an insufficient but necessary part of a condition which is itself unnecessary but sufficient for the result », (p. 245). 
pour Simon, purement conventionnelle et par conséquent arbitraire. La distinction dépend de ce qui dans une situation expérimentale est considéré comme manipulation ou contrôle de l'expérimentateur (condition ou variable indépendante) et de ce qui n'est pas manipulé (proposition d'observation ou variable dépendante). Simon a tenté de résoudre ce problème de l'asymćtrie arbitraire en définissant axiomatiquement, sur un système d'équations linéaires (où figurent les variables des supposées relations causales), des «self-contained structures ${ }^{6}$, c'est-à-dire un ordre dans les équations, dont la hiérarchie indique l'ordre causal des variables. Il s'agit d'une situation où l'information à propos des données empiriques peut être représentée par un ensemble d'équations algébriques linéaires qui forment un système à structure linéaire. Simon propose alors une façon de résoudre ces équations qui, du point de vue de l'algèbre au moins, ne fasse pas appel à la distinction entre variables dépendantes et variables indépendantes, car toutes les variables sont interdépendantes. Il s'agit dans cette solution d'isoler des sous-ensembles d'équations, allant du plus petit ( degré $O »$ ) au plus grand ( degré $\mathrm{n} »$ ), qui puissent être résolus pour chaque degré sans recourir à des équations d'un degré supérieur. Les degrés indiquent la hićrarchie des variables et celle-ci livre l'ordre de causalité, ordre qui garantit l'asymétrie des variables, ceci sans recourir à de l'information supplémentaire concernant p.ex. la séquence temporelle. Pour Simon, un tel système d'équations possède des propriétés structurelles en vertu desquelles les variables forment une hiérarchie. C'est donc à ce type de structure formelle qu'est ramenće l'idée de causalité.

Le plus nuancé des défenseurs de la causalité dans les sciences sociales est certes Blalock ' ${ }^{\top}$ Ses réflexions critiques touchent moins le problème de la signification, (que signifie l'expression: «a cause $\mathrm{b} » ?)$, que le problème de la v'érification des propositions causales, surtout dans des situations non expérimentales où l'influence des variables exogènes ne peut pas être

6. SIMON, H.A., op. cit., «Causal Ordening and Identifiability», pp. 10-37.

7. BLALOCK, H.M., Jr. Causal Inferences in Non-Experimental Research, Chapel Hill (The University of North Carolina Press), 1964; et BLALOCK, H.M., Jr. (ed.), Causal Models in the Social Sciences, Chicago (AldineAtherton), 1971. 
neutralisée et où l'expérimentateur manque d'information, p.ex. au sujet de la séquence temporelle. Blalock tient les propositions causales pour des hypothèses de travail qui lui paraissent très utiles dans une optique pragmatique de la science; mais elles ne pourraient pas être soumises à un contrôle empirique. D'une part, il existe, d'après Blalock, un écart trop grand entre les instruments formels permettant d'exprimer mathématiquement les résultats de l'expérience et de l'observation et le langage causal qui implique, d'une manière ou d'une autre, la notion de "production » qui n'est guère formalisable ou traduisible dans ce langage formel. D'autre part, l'asymétrie que sous-entend lidée de production connote plus que l'asymétrie temporelle.

Blalock aborde aussi le problème de la contingence. Si l'on interprète une corrélation positive entre deux variables, soit la «densité de la population» (a) et le «taux de criminalité » (b), comme une relation de cause à effet, il peut toujours s'agir d'une relation contingente ou accidentelle ( $\ll s p i$ rious ») qui disparait dès qu'on introduit une troisième variable, par.ex. «niveau de revenu » (c) ${ }^{\varepsilon}$. Mais si on définit l'expression : $[\mathrm{r}(\mathrm{a}, \mathrm{b})$ si non accidentelle avec $\mathrm{r}(\mathrm{a}, \mathrm{b} ; \mathrm{c}) \rightarrow$ « a est cause de $\mathrm{b} \gg]^{9}$, on peut toujours invoquer de nouvelles variables qui rendront, le cas échéant, la (ou les) relation(s) précédente(s) contigente(s), de sorte qu'une hypothèse causale ne pourrait jamais être réfutée et se trouverait être en principe non falsifiable; elle est donc une affirmation dogmatique, ce que Glock et Hyman avouent sans ambages: "...actually, there is no way with survey data guarantee that a relationship is causal ${ }^{10}$ et «...all explanations are spurious...; ...in practice one can

8. 'Dans ce cas on obticnt soit une interprétation, c'est-à-dire que a n'est pas la cause directe de $b$ mais agit par l'intermédiaire de $c(a \leftarrow c \rightarrow b)$, soit une «explication» («explication» en anglais), à savoir que c'est la cause de $a$ et de $b$ sans aucune relation entre a $c t \quad b \quad(a \leftarrow c \rightarrow b)$. Le problème de la «spuriousness» a surgi au moment où on a constaté en Angleterre une corrélation positive très élevée entre «taxe de téléphone» ct «niveau d'imbécilité», ce qui ne pouvait signifier: "plus vous téléphonez, plus vous avez la chance de devenir imbécile».

9. LAZARSFELD, P., PASANELla, A.K., ROSENBERG, M., (cds.), Continuities in the Langulage of Social Research. New York (The Free Press), 1972 , p. 121.

10. GLOCK, Ch. Y., Surcey Research in the Social Sciences, Now York (Russel Sage), 1967, p. 21. 
never reach the original cause in the developmental sequence..., the logic of spuriousness becomes an absurdity ${ }^{11}$. Et Lazarsfeld conclut: "Empirically, we will never definitively establish a causal relation ${ }^{12}$. L'analyse causale reste un programme. Le mieux qu'on puisse faire c'est construire des modèles «which make use of a whole serie of untestable simplifying assumptions ${ }^{13} \gg$

Trois groupes de sociologues s'opposent à l'analyse causale : les néo-fonctionnalistes, les ethnométhodologistes et certains structuralistes. Le fonctionnalisme, devenu classique dans. les sciences sociales avec Radcliff-Brown, Durkheim et Talcott Parsons, qui opèrent dans les limites de l'analyse causale, ramène le concept de «cause» soit à celui de «cause efficiente», soit à celui de «cause finale», concepts qui ont des connotations proprement philosophiques. Mais la reformulation de quelques principes du fonctionnalisme par Luhmann ${ }^{14}$ semble prendre ses distances vis-à-vis une telle réduction. Dans l'optique classique, une composante d'un système d'action remplit une fonction dans la mesure où elle contribue au maintien d'un état de système, au maintien d'un processus dans un état d'équilibre ou à la restauration d'un état de système. Ces « effets» s'obtiennent grâce à des dispositions latentes que tout système social est censé posséder, dispositions qui, dans le cas de perturbations, sont activées et contribuent, en tant que «causes» ou combinaisons complexes de relations causales, au maintien du système. Ce schéma causal inhérent à l'analyse fonctionnelle n'a pas résisté à la critique ${ }^{15}$. Ceci a amené les néo-fonctionnalistes,

11. HYMAN, H., Survey Design and Analysis, Principles Cases Procedures, Illinois (The Free Press), 1955, p. 255.

12. LAZARSFELD, P. et al., op. cit., p. 121.

13. BLALOCK, H., Causal Inferences, op. cit., p. 6.

14. LUHMANN, N., «Funktion und Kausalität» et «Funktionale Methode und Systemtheorie» in: LUHMANN, N., Soziologische Aufklarung, Aufsätze zur Theorie sozialer System, Köln/Opladen (West deutscher Verlag) 1970; LUHMANN, N., Zweckbegriff und System rationalität, Tübingen (Mohr), 1968.

15. Notamment les critiques de NAGEL, E., Logic Witbout Metaphysics, Glencoe III, (The Free Press), 1958, pp. 247 ss. NAGEL, E., The Structure of Science, Problems in the Logic of Scientific Explanation, New York (Harcourt, Brace and World Inc.), 1961, pp. 520 ss. HEMPEL, C., "The Logic of Functional Analysis», in: GROSS, L. (ed.) Symposion on Sociological Theory, Evanston III - White Plains New York (Row, Peterson), 1959, pp. 271-307. 
dont Luhmann, à déplacer la question. Au lieu de s'intéresser à des fonctions comme des causes déterminées qui entraînent des effets déterminés, on s'intéresse aux «équivalences fonctionnelles », c'est-à-dire à des solutions alternatives pour un même problème du système. Le terme de «cause » désignera un ensemble d'événements (actions) fonctionnellement équivalents pour la réalisation d'une conséquence déterminée, et le terme "effet » désignera un ensemble d'événements (conséquences) fonctionnellement équivalents par rapport à une action déterminée ${ }^{16}$. De ce fait, les questions concernant la causalité sont subordonnées aux questions relatives aux structures des équivalences fonctionnelles.

Ceux qui s'opposent le plus violemment au concept de «cause»dans les sciences sociales sont les tenants de la sociologie compréhensive ou de l'ethnométhodologie ${ }^{17}$. Il est trop simple de récuser leur point de vue en déclarant que la «méthode » de compréhension ne fournit qu'un contenu de connaissance trivial. Leur argument parait plus solide. Il repose sur des différences touchant la nature des relations entre les phénomènes sociaux, c'est-à-dire entre les actions. Puisque l'action est un comportement doué de sens, un système d'action est un ensemble de relations significatives et non pas un ensemble de relations causales. L'analyse interprétative ou herméneutique se propose d'expliciter la structure intentionnelle de la signification d'une action. Les herméneuticiens reprochent aux «causalistes»de manquer les propriétés clefs d'une action aussi longtemps qu'ils écartent, au nom de la nature de la relation causale, l'aspect intentionnel de l'action.

Le troisième groupe qui s'oppose à l'analyse causale est formé par les structuralistes. Mais ceux-ci ne font pas l'unanimité dans leur camp. Les althussériens, d'une part, rejettent la causalité linéaire au profit d'une nouvelle forme de causalité qui serait requise pour rendre compte des phénomènes économiques, à savoir la «causalité structurale». Dans cette optique,

16. LUHMANN, N., Zueckbegriff und Systemrutionalitait, op. cit., ch. 1, pp. $9 \cdot 36$.

17. Les représentants principaux sont: SCHÜTZ, A., Collected Papers, 3 volumes, The Hague (Martinus Nijhoff), 1962-1966. HABERMAS, J., Zur Logik der Sozialuissenschaften, Frankfurt (Suhrkamp) 1970; et GARFINKEL, H., Studies in Etbnometbodology, New Jersey (Prentice-Hall, Inc.), 1968. 
la structure est elle-même prise pour une cause; elle a une efficace, elle exerce une action sur ses propres éléments. Cette efficace de la totalité sur les parties est paradoxalement une cause absente, puisque l'existence de la structure qu'il faut postuler consiste dans ses effets; mais en tant quarticulation même de ses effets, elle ne peut être elle-même située parmi ces effets; elle est donc absente ${ }^{18}$. Les structuralistes moins spéculatifs, d'autre part, ne peuvent pas se résigner à ce statut paradoxal et à l' «ontological commitment» quant à l'existence d'une structure et quant à son pouvoir causal. Ces structuralistes semblent présenter un type d'analyse de la réalité sociale, qui prétend appréhender celle-ci dans des aspects fondamentaux, qui prétend atteindre le niveau des structures profondes par opposition à ces manifestations superficielles. À ce niveau-là, il n'y aurait plus de place pour des «causes» et la catégorie de la causalité deviendrait tout simplement superflue.

C'est dans cette dernière optique que nous pourrions interroger les travaux de Lévi-Strauss dont l'analyse structurale des systèmes de parenté peut être considérée comme paradigmatique. La question qui va nous guider est la suivante: si on réussit à exhiber une structure convenable ou à fournir une explication structurale des phénomènes sociaux, ne reste-t-il effectivement plus aucune question plus fondamentale, qui concernerait, p.ex. les «causes» de l'évolution des structures? Le traitement structural permet-il de résorber l'analyse causale et rend-il celle-ri redondante?

\section{Le structuralisme de Lévi-Strauss}

L'analyse de système peut être menée sur deux plans, celui des processus et celui des architectures. L'étude des systèmes de parenté de Lévi-Strauss ${ }^{19}$ s'en tient au dernier. Pour Lévi-Strauss, les systèmes de parenté constituent un univers formé d'ensembles organisés conformément à des lois de nature combinatoire. Cette façon de traiter des systèmes de parenté n'exclut pas absolument l'aspect de processus puisqu'on peut également envi-

18. AlTHUSSER, L., BALiBAR, E., Pour lire le Capital II, Paris, (Maspero, Petite Collection), pp. 56-71.

19. LÉVI-STRAUSS, Cl., Les structures élémentaires de la parenté, Paris/ La Haye (Mouton), 1968. 
sager le passage d'une configuration de système à une autre configuration. Mais il semble que ces «processus» soient d'une autre nature que les simples changements d'état. Il ne s'agit pas de processus évolutifs (où il y aurait éventuellement place pour la causalité) en tant que succession réglée d'états de système dans le temps, mais de processus métamorphiques en tant quautostructuration d'une structure, processus qui pourrait luimême obéir à des lois de structure. Le langage approprić pour se représenter les architectures est l'algèbre, notamment l'algèbre des matrices moyennant laquelle Weil ${ }^{20}$, Bush ${ }^{21}$, Kemeny, Snell et Thompson ${ }^{22}$ ont reformulé les hypothèses centrales des travaux de Lévi-Strauss. L'algèbre des matrices donne à l'étude structurale une forme plus rigoureuss. Elle est utilisée dans bien d'aurres domaines de la sociologie où l'approche structurale s'est avérée féconde ${ }^{2: 3}$. Il s'agit de toute une série de travaux fort significatifs qui procèdent par la méthode structurale et q!ii n'ont apparemment pas besoin du concept de «cause ».

20. Appendice à la première partic de Structures clémentaires de la parenté, pp. 257-270.

21. Extension de lappendice de WEIL: «An Algebric Treatment of Rules of Marriage and Descent», in: WHITE, H.C., An Analysis of Kinship, Now Jersey (Prentice-Hall, Inc.), 1963, pp. 159-173. (Pout la date de publication de larticle de Bush voir KORN, F., Elementary Structures Reconsidered, Lćvi-Strauss on Kinship, Berkeley/Los Angeles (University of California Prcss), 1973, p. 126, note 2.).

22. KEMENY, J.G., SNELL, J.L., THOMPSON, G.L., Introduction to Finite Mathematics, Englewool Cliffs, New Jersey (Prentice-Hall), 1957.

23. En sociométric, HARARY, F., ROSS, I.C., «A Procedure for Clique Detection Using the Group Matrix », in: Socionetry 20, 1957, pp. 205.215; LUCE, R.D., PERRY, A.D., «A Method of Matrix Analysis of Group Structure», in: Psycbometrica 15, 1950, pp. 169-190 et FESTINGER, L., «The Analysis of Sociograms Using Marrix Algebra», in: Human Relations 2, 1949, pp. 153-158, étudient les groupes en vue de détecter par l'analyse matricielle la structure de clique. BLAL, P., Burocracy in Modern Society, New York (Random House, Inc.), 1956; LUHMANN, N., Fanktionen und Folgen Formaler Organisation, Berlin, (Dunker und Humblot), 1964, approchent les organisations formelles par les mêmes instruments et constatent, notamment Blau, une très grande analogie entre les arbres génćalogiques et les «arbres de rôles» dans un système bureaucratique; BALES, R.F., et al. "Channcls of Communications in Small Groups », in : Ancricun Sociological Retieu 15, 1951, pp. 461-468, ct BAVELAS, A., "A Mathematical Model for Group Structures», in: Applicd Antbropology 7, 1948, pp. 16-28 dégagent des proprićtés formelles des systèmes ou des réseaux de communication, de même que FLAMENT, F., Réseaux de communication et structures de groupe, Paris (Dunod), 1965. 
Quand on se place dans la perspective des recherches signalées ci-haut, on peut apprécier la portée des analyses de Lévi-Strauss. Il devient alors clair que la tentative de celui-ci fut de construire (de définir) des structures sur un ensemble d'éléments (les termes de parenté), et ceci moyennant un certain nombre d'axiomes qu'il n'a pourtant jamais explicitement formulés en vue d'un traitement formel. Pour une meilleure compréhension on les donnera ici in extenso en recoupant les formules de Bush ${ }^{24}$, de White ${ }^{25}$ et de Boudon ${ }^{26}$. Ils forment les postulats d'une théorie générale de la parenté (avec certaines restrictions précises).

Ax.1 La population de chaque système de parenté est considérée comme réductible à des clans mutuellement exclusifs. Plus formellement: est donné un ensemble $\mathrm{K}$ avec $\mathrm{n}$ sousensembles $F_{1} \ldots$. Fn de sorte que $F_{1} \frown F_{2}=\phi$, chaque élément (individu) appartenant à un et un seul sousensemble (clan).

Ax.2 Dans chaque système de parenté (ensemble), il existe une règle permanente qui détermine le clan (sous-ensemble) unique où les membres d'un autre clan déterminé doivent trouver leurs partenaires. Plus formellement: il existe un opérateur de permutation qui transforme chaque point en un autre point.

Ax.3 Les hommes de clans (sous-ensembles) différents ne peuvent pas prendre pour partenaires des femmes appartenant à un même clan (sous-ensemble).

Ax.4 Tous les descendants d'un couple sont assignés à un clan (sous-ensemble) unique déterminé seulement par les clans (sous-ensembles) auxquels appartenaient leurs pères ou leurs mères.

Ax.5 Les descendants dont les parents appartiennent à des clans différents appartiennent eux-mêmes à des clans (sousensembles) différents.

24. BUSH, R., op. cit, pp. 159-72.

25. WHITE, H.C., op. cit., ch. II.

26. BOUDON, R., A quoi sert la notion de structure, Essai sur la signification de la notion de structure dans les sciences humaines, Paris (Gallimard), 1968. 
Ax.6 Un homme ne peut jamais marier une femme de son propre clan. (Prohibition de l'inceste).

Ax.7 Chaque membre du système de parenté a un parent par alliance ou par descendance dans chaque autre clan (ce qui veut dire que le système ne peut pas se partager en groupes qui ne seraient pas liés à tous les autres).

Il semble que ces axiomes soient suffisants pour rendre compre de tous les systèmes élémentaires ${ }^{27}$ de parenté. Il reste à remarquer que ces axiomes ont un statut ambigu, car certains constituent des règles dans le sens de normes ou de prescriptions, lesquels peuvent sûrement être traduits en termes de normes conscientes. Ce n'est pas le cas pour tous. Si, p.ex., Ax.1 stipule une partition en $\mathrm{n}$ sous-ensembles (clans), il n'est pas requis qu'on obtienne une évidence empirique pour ce nombre ou que les indigènes possèdent des prescriptions quant à cette répartition. Il arrive que la classification qu'effectuent les membres indigènes d'un système de parenté ne correspond pas à la classification qui est théoriquement requise pour rendre compte de l'intégralité des phénomènes observés ${ }^{28}$.

Dans un système formel (ou «modèle» dans le langage de I.évi-Strauss), le terme de «structure» désigne une classe de conditions, exactement les conditions formelles qui déterminent la distribution des relations pour l'ensemble des combinaisons permises d'éléments ou de signes. Mais, comme ces relations sont le résultat d'un certain nombre d'opérations qu'on peut effect:ıer sur les éléments (qui peuvent rester indéterminés et dont la nature est elle-même sans importance pour l'analyse structurale), la notion de «structure» réfère aux conditions imposées aux opérations à effectuer dans le système. On a par conséquent pour le terme de «structure», pris au sens général, la définition suivante: «structure»= déf. «la classe de toutes les classes

27. Pour une définition de «eystème élémentaire», voir LÉVI-STRAUSS, Cl., Les structures élémentaires de la parenté, op. cit., pp. IX ss. ou la discussion de KORN, F., Elementary Structures, op. cit., ch. 2, ou de NEEDHAM, R., Structures and Sentiment, Chicago (University of Chicago Press), 1962, ch. 1.

28. Ceci est démontré par André WEIL, op. cit. à propos du système Murngin.

29. LÉVI-STRAUSS, Cl., Antbropologie structurale, Paris (Plon), 1958, pp. 284-285. 
de conditions définies sur des opérations ${ }^{30}$. Il s'ensuit que le concept de «structure » doit être considéré comme un concept métathéorique. Cette définition de la structure veut clairement mettre en évidence le caractère formel du concept en question; elle devrait nous tenir éloignés d'une interprétation ontologique qui hypostasie les entités formelles. Ce point sera discuté dans la troisième partie.

Quelles sont maintenant les opérations qu'on invoque quand on aborde les relations de parenté et de quelle manière les axiomes formulés ci-hauts constituent-ils les conditions imposées à ces opérations? Si d'après Ax.1 la population est réductible à $\mathrm{n}$ clans, où $\mathrm{n}=[1,2,3,4]$, la règle dans $A x .2$ peut être représentée, comme le suggèrent Kemeny, Snell, Thompson, Bush et White, par un opérateur mathématique, l'opérateur de permutation de côté $n$, ce qui est une matrice carrée; les rangs de cette matrice correspondent aux clans des hommes, les colonnes aux clans des femmes. Cette matrice carrée ne contient que des $\langle I »$ et des « $O$ » conformément aux restrictions qu'impose Ax.1; elle montre le clan dans lequel une femme de n'importe quel clan donné peut chercher son partenaire, ou inversement le clan dans lequel un homme de n'importe quel autre clan peut chercher sa partenaire ${ }^{31}$. Pour la société Kariera que LéviStrauss a étudiée, la matrice serait:

$$
F=\left[\begin{array}{llll}
O & I & O & O \\
I & O & O & O \\
O & O & O & I \\
O & O & I & O
\end{array}\right]
$$

Un opérateur analogue peut être donné pour représenter la règle de descendance (Ax.4). De nouveau, pour la sociétć Kariera avec ses quatre clans, la matrice de descendance serait:

$$
\mathrm{D}=\left[\begin{array}{cccc}
\mathrm{O} & \mathrm{O} & \mathrm{I} & \mathrm{O} \\
\mathrm{O} & \mathrm{O} & \mathrm{O} & \mathrm{I} \\
\mathrm{I} & \mathrm{O} & \mathrm{O} & \mathrm{O} \\
\mathrm{O} & \mathrm{I} & \mathrm{O} & \mathrm{O}
\end{array}\right]
$$

30. La définition donnće correspond à celle de BOURBAKI, N., Théorie des ensembles, Paris (Hermann), 1964, 8.2; les structures d'une espèce déterminće appelées «anneaux », 《groupes », «monö̈des», etc., réfèrent de nouveau à un nombre de proprićtés - une classe déterminée de conditions - d'un nombre d'opérations à effectuer sur des ćléments.

31. Voir WHITE, H.C., op. cit., p. 35. 
Par la matrice $\mathrm{F}$ on passe du clan de n'importe quel homme au clan de n'importe quelle femme et par la matrice $D$ on passe du clan de n'importe quel parent au clan de n'importe quel enfant ${ }^{32}$.

Comme on peut le voir aisément, pour une séquence donnée de $\mathrm{N}$ nombres, il existe $\mathrm{N}$ ! possibilités d'opérateurs de permutation pour $\mathrm{F}$ seulement et $(\mathrm{N} !)^{2}$ pour $\mathrm{F}$ et $\mathrm{D}$ combinés. Ceci met bien en évidence la puissance de cet instrument mathématique qui permet de générer, au moins sur le plan formel, un grand nombre de systèmes (de parenté en occurrence). Mais beaucoup de ces permutations possibles violent les axiomes donnés et ces opérateurs ne peuvent dès lors pas être retenus. Par exemple, une des N! matrices possibles pour F est l' «opérateur d'identité » qui transforme une séquence $[1,4,3,2$,$\} en une séquence iden-$ tique; un tel opérateur est exclu en vertu de Ax.6. En éliminant aussi des opérateurs qui sont structurellement analogues, on obtient finalement un nombre limité d'opérations admissibles qu'on fera correspondre aux règles d'alliance et de descendance des différentes sociétés à structure élémentaire de parenté.

Comme matrices, $\mathrm{F}$ et $\mathrm{D}$ peuvent également être combinées par la multiplication des matrices. Le produit de matrice $F \times D$ spécifie, an langage de parenté, pour un homme de chaque clan le clan auquel appartient l'enfant du frère de sa femme. Toute suite ordonnée et toute puissance de $\mathrm{F}$ et $\mathrm{D}$ donnent une nouvelle matrice de permutation spécifiant pour chaque clan possible d'un homme le clan d'un de ses parents. En d'autres termes, deux matrices et leurs combinaisons (applications successives) montrent comment on passe de n'importe quel point du système à n'importe quel autre point pour savoir quel sera le clan auquel appartient le second élément étant donné le clan du premier, et vice versa.

La question principale à poser maintenant est la suivante: pour une société déterminée avec $\mathrm{F}$ et $\mathrm{D}$ données (par hypothèse), quelle relation peut être une relation d'alliance? En termes de l'anthropologue ou de l'ethnographe, les phénomènes simples qu'il faut expliquer adéquatement ou les questions clefs sont: pourquoi n'y a-t-il pas de lien d'alliance entre cousins parallèles

32. Ibidem, pp. 36-37. 
(de Ego avec la fille de la soeur de la mère ou du frère du père) ? Pourquoi y a-t-il alliance entre cousins croisés et de préférence entre cousins croisés matrilatéraux? Ce sont ces faits observés qui doivent être expliqués, et les anthropologues semblent admettre que Lévi-Strauss ne fournit pas seulement une description cohérente des phénomènes en question, mais l'explication qu'ils cherchaient. Cette explication répond à la question: à partir de quelles lois et de quelles conditions initiales peut-on déduire les faits observés? Dans ce sens elle satisfait les exigences d'une «H-D-explanation» de Hempel ${ }^{33}$. En effet, le fait qu'il n'y a pas d'alliance, p.ex. entre cousins parallèles matrilatéraux, est strictement déductible des axiomes considérés comme des hypothèses nomologiques et de conditions initiales observées. Voici le schéma qu'on obtient en suivant White ${ }^{34}$ :
[1] $\mathrm{M}=\mathrm{D}^{-1} \mathrm{~F} \quad \mathrm{~F}^{-1} \mathrm{D}$
$M$ est une matrice correspondant à un produit de matrice, qui indique le chemin de A à $B$, c'est-à-dire le che- $\min$ entre les cousins en question ${ }^{35}$.

\section{[2] $\mathrm{M}=\mathrm{D}^{-1} \mathrm{D} \quad \mathrm{F}^{-1} \mathrm{~F}$ \\ [3] $\mathrm{I}=\mathrm{D}^{-1} \mathrm{D} \quad \mathrm{F}^{-1} \mathrm{~F}$}

[4] $\mathrm{M}=\mathrm{I}$

[5] $\mathrm{M}=\mathrm{F}$

[6] $\mathrm{F}=\mathrm{I}$
Loi associative démontrée en algèbre des matrices, I étant une matrice d'identité.

de [2] et de [3].

On met par hypothèse que $M$ est une matrice d'alliance, $F$.

De [4] et [5], ce qui contredit Ax.6 selon lequel un homme ne peut pas marier une femme de son propre clan. L'hypothèse [5] mène à une proposition contradictoire. Elle doit être rejetée et par conséquent il n'y a pas

33. HEMPEL, C.G., Aspects of Scientific Explanation, An other Essay in the Philosophy of Science, New York (The Free Press), 1968, Ch. IV.

34. WHITE, H.C., op. cit., p. 40.

35. Schéma : $\Delta_{\bar{\delta}} \delta \overline{\mathrm{J}}^{\Delta}$.

Du clan de l'entant $A$ on remonte au clan da père par l'inverse de la matrice $\mathrm{D}$ (matrice de descendance), donc $\mathrm{D}^{-1}$; du clan du père au clan de la mère par la matrice $F$ qui spécifie aussi le clan de la soeur de la mère, et de là, par $\mathrm{F}^{-1}$, on passe au clan du mari de la soeur de la mère et, finalement, par D au clan de l'enfant B. 
d'alliance entre cousins parallèles matrilatéraux, fait qui est maintenant formellement expliqué, ou, comme Boudon le dit, «... il se trouve que l'interdiction du mariage entre cousins parallèles est maintenant une conséquence générale de l'axiomatique ${ }^{36}$. On répond de la même manière aux autres questions.

\section{Le concept de cause est-il relayé par le concept de structure?}

Certains pourraient croire que les «modèles» que construit Lévi-Strauss pour des systèmes de parenté sont une affaire de taxonomie; il s'agirait par conséquent seulement d'une classification, d'une typologie des diverses sociétés, ou d'une description plus systématique qui codifierait la variété des observations ethnographiques fort hétérogènes. On peut admettre, avec la majorití des critiques récents qui examinent principalement l'aspect épistémologique du structuralisme, que Lévi-Strauss fait autre chose. Analogues à l' «Idealtypus » de Max Weber, dont Hempel a clarifié le statut ${ }^{3 \tau}$, les «modèles» de Lévis-Strauss ne constituent pas des typologies classificatoires ou descriptives, mais ils représentent de véritables systèmes théoriques. Bien que construits à l'aide d'instruments mathématiques, - ce qui permet le passage à la «structure profonde», entité théorique pour laquelle on postule des propriétés non observables, - les «modèles » ne peuvent pas être considérés comme des systèmes purement formels, car les éléments qui y figurent sont interprétés en termes de «membre d'un clan», «alliance», «descendance», etc., qui doivent passer pour des termes d'observation. De la sorte on rend compte, moyennant la construction théorique, des phénomènes observables. Dans ce sens, les «modèles» ont une portée empirique ${ }^{38}$. Ils ont en plus un pouvoir explicatif, comme le dit Lévi-Strauss tout au long de ses recherches, principalement dans un passage où il s'explique lui-même sur la portée explicative de ses $«$ modèles ${ }^{39}$. Si une explication est une réponse à un «Pourquoi?" " c'est-à-dire une réponse à un «uell-formulat-

36. BOUDON, R., À quoi sert la notion de structure, op. cit., p. 136.

37. HEMPEL, C.G., Aspects of Scientific Explanation op. cit., p. 161 ss.

38. Cf. LÉVI-STRAUSS, C., Antbropologie structurale, op. cit. ch. II et ch. XV et «Sens et usage de la notion de modèle», in: Anthropologie siructurale deux, Paris (Plon) 1973, pp. 89-105.

39. LÉVI-STRAUSS, C., Antbropologie structurale, op. cit., p. 306 ss. 
ed problem ${ }^{40}$ comme : «à partir de quelles lois et de quelles conditions initiales la proposition d'observation $S$ peut-elle être déduite?»), c'est bien ce que fait Lévi-Strauss comme on l'a montré ci-haut. En effet, les phénomènes observés sont les conséquences qui découlent des hypothèses concernant les propriétés des structures sous-jacentes.

Mais, comme on l'a déjà noté, les hypothèses en question ont un statut ambigu. Toutes ne sont pas des hypothèses nomologiques et ne constituent dès lors pas de véritables lois; certaines ont un caractère clairement normatif (cf. Ax.3, Ax.6, $A x .2)$. L'ensemble des axiomes pourrait être un système de règles qui seraient difficiles à réduire à des hypothèses, puisque l'existence et la contrainte propre à une règle sont contingentes tandis que la contrainte propre à une loi est (le plus souvent considérée comme) nécessaire. C'est exactement dans ce sens que Talcott Parsons entend définir la structure sociale quand il écrit : «La structure sociale d'un système social ... consiste en un pattern institutionnalisé de culture normative ${ }^{41}$. L'étude des systèmes de parenté peut porter sur des règles qui auraient un triple statut :

a) On peut les prendre pour des règles d'action explicites, s'adressant à des acteurs spécifiques, donnant ainsi lieu à des attentes mutuelles, justifićes par la conformité d'autres acteurs à l'égard de ces règles.

b) Elles peuvent être des régularités induites à partir des corrélations statistiques des mariages entre certaines catégories d'individus. Elles auraient dans ce cas à peu près le même statut que les règles de la «grammaire profonde » de Chomsky; elles seraient des règles que personne ne promulgue, ni ne sait, ni ne suit consciemment.

c) Ou bien ce sont des règles qui président aux arrangements des termes de parenté, car ces arrangements peuvent contenir implicitement des «impératifs » ou des prescriptions pour un comportement déterminé. Les «modèles de

40. BUNGE, M., Scicntific Research II, The Search for Truth, Berlin-HeidelbergNew York (Springer-Verlag), 1967, p. 12.

41. PARSONS, T., "An Outline of the Social System», in: PARSONS, ' $T$., SHILS, E., NAEGELE, K., PITTS, J.R., Theories of Society, New York (The Free Press), 1965, p. 36. 
Lévi-Strauss sont probablement une combinaison, comme pense Korn ${ }^{42}$, de ces trois sortes de tègles $»$. Ou, dans les termes de Braybrooke «They may be rules that express the expectations of the people that they are ascribed to; and these people may in fact conform to them, but they may not be rules that those people ball ever formulated for themselves $\gg{ }^{43}$.

Il semble donc que l'analyse des systèmes de parenté de Lévi-Strauss oscille ${ }^{\$ 4}$ entre un ensemble d'axiomes formulés dans une logique des normes (au sens de von Wright), faisant intervenir expectation et conformité, et un ensemble d'axiomes formulés dans une logique de l'action, énonçant la régularité de certaines actions sans faire appel à des expectations et à la conformité ${ }^{45}$. La question est maintenant de savoir si les axiomes formulés dans une logique des normes peuvent figurer comme prémisses dans une explication hempelienne. Braybrooke le pense quand il affirme que les règles "may reasonably serve in place of the lawlike premises called for in Hempel's basic nomological pattern of explanation ${ }^{46}$. Mais pour cela Braybrooke devait tout d'abord, suivant von Wright, montrer qu'une règle admet une sorte de quantification, qu'elle a une certaine généralité eu égard aux occasions d'application possibles et eu égard à la classe d'acteurs visés par la règle. À cela on peut objecter que la généralité ne signifie pas l'universalité requise pour l'explication hempelienne, et qu'on ne peut pas conclure, à partir de l'existence d'une règle, que $\mathrm{X}$ exécute effectivement l'action prescrite (telle qu'elle pourrait être décrite par une proposition d'observation) mais que $\mathrm{X}$ devrait la faire, comme Stegmiller l'a analysé ${ }^{47}$. Si, par contre, on traduit les prémisses normatives en lois empiriques (pseudo-empirique?) concernant le comportement d'observance d'une règle, il restera toujours que de telles lois

42. KORN, F., Elementary Structures Reconsidered; op. cit., p. 144.

43. BRAYBROOKE, D., «Refinements of Culture in Large-Scale History", in : Theory and History, Beinheft? 1969, p. 56.

44. L'ambiguïté qu'on signale ici donne lieu chez Lévi-Strauss au dédoublement de «modèle conscient» et «modèle inconscient», cf. LÉVI-STRAUSS, C., Antbropologie structurale, op. cit., p. 308.

45. Notons que «conformité » est déjà un terme évaluatif.

46. BRAYBROOKE, D., «Refinements of Culture», op. cit., p. 61.

47. STEGMÜLLER, W., Probleme und Resultate der Wissenschaftstbeorie und Analytischen Pbilosophie, Band I Wissenschaftliche Erklärung und Begründung, Kapitel VI. 7, «Rationale Erklärung》, pp. 379-398. EerlinHeidelberg-New York (Springer-Verlag), 1969. 
contiendraient des restrictions quant aux occasions d'application de la règle; elles ne seraient par conséquent pas strictement universelles et cela entraînerait des conséquences indésirables, notamment que l'explication ainsi obtenue ressemblerait étrangement à une explication ad boc, comme Popper le signale ${ }^{48}$.

À supposer qu'on ait résolu les problèmes soulevés à propos de l'explication chez Lévi-Strauss, celle-ci constitue-t-elle une explication causale? Certainement pas, à moins qu'on ne définisse l'expression « $a$ cause $b$ » par «b suit logiquement de a et de lois ». Mais dans ce cas, ou bien toute explication passera pour être causale, ou bien on exige que les lois en question soient des lois causales (dont la forme ne peut ètre précisée) et alors la définition est circulaire. De plus, le schéma hempelien que la définition résume est symétrique; $a$ et b sont interchangeables, ce qu'on ne peut guère admettre pour une relation causale. Voyons si la distinction de Hempel entre «lois de succession» et «lois de coexistence» nous permet de sortir de l'impasse ${ }^{40}$.

Une explication causale requiert généralement des lois de processus. Il est possible d'identifier dans les systèmes de parenté des états de systèmes qui se succèdent; les états en question correspondraient aux générations qui se suivent. Il faut admettre que l'ordre dans les relations d'alliance d'une génération est fonction des alliances de la génération précédente, ce que LéviStrauss affirme pour des sociétés à «structure élémentaire». La succession dont il est question est la transformation réglée d'une séquence initiale d'appartenance au clan:

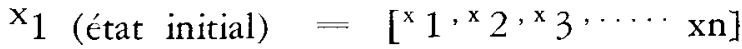

$\mathrm{x}_{2}$ (état suivant) $=\left[\begin{array}{c}{ }^{\mathrm{x}} 1 \\ { }^{\mathrm{x}} 2 \\ \cdot \\ \cdot \\ \cdot \\ \cdot \\ \mathrm{x}\end{array}\right]=\{$

48. POPPER, K.R., The Logic of Scientific Discovery, New York, (Harper Torchbooks), 1965, 13, pp. 62 ss.

49. HEMPEL, C.G., Aspects of Scientific Explanation, op. cit., pp. 347-352, et «Deductive-Nomological Explanation vs Statistical Explanation», in : FEIGL, H., MAXWELL, G., (eds.) Minnesota Studies in the Philosophy of Science, vol. III, Minneapolis (University of Minnesota Press), 1962, p. 108. 
(où F.D, ou tout autre produit ou puissance des deux matrices, est un opérateur de permutation). Il s'agit bien d'une suite d'espaces de configuration, d'une sorte de transmission « héréditaire », mais tous les états successivement réalisables (les générations) sont plutôt les manifestations d'une même loi de distribution. Les axiomes de Lévi-Strauss ne sont pas des lois de processus, ce sont des lois de coexistence ou de confirguration; à tout le moins ne décrivent-elles pas le passage d'une structure à une autre. Lévi-Strauss ne peut par conséquent pas considérer l'explication qu'il propose comme une explication causale. Il s'agit d'une explication structurale qui consiste à dégager des types de récurrences significatives qui sont plus générales que des causalités singulières $\gg .{ }^{50}$

Si on veut introduire des «causes» dans un tel schéma explicatif, on peut prendre les «modèles» que construit LéviStrauss pour des simulations d'une réalité, pour un ensemble d'opérations par lesquelles le savant tente de réeffectuer pour son propre compte une réalité particulière. Il est alors possible d'interpréter l'opérateur de permutation (FD) comme une cause qui effectuerait véritablement la distribution des partenaires d'une génération à l'autre. On pourrait faire correspondre l'opérateur en question à une opération inconsciente effectuée par l' «esprit indigène » et donnant lieu aux effets observés. Mais il faudrait alors expliquer en même temps comment cet «opérateur» se trouve dans l'inconscient des indigènes, comment il avait été intériorisé (pour le cas où on voudrait recourir à une thécrie de l'apprentissage) et, avec lui, tout le système des axiomes qui constituent ses conditions limitatives. Il est également possible de considérer l'opérateur en question comme la simple formulation d'une règle d'action et de se demander si, le cas échéant, une règle agit comme une cause. D'habitude les règles constituent les raisons qu'on a pour agir dans un sens déterminé; on les mentionne au moment où il faut justifier sa propre action. Prétendre que ces raisons agissent comme des causes (au sens de INUS-conditions de Mackie) ${ }^{51}$, à condition que l'acteur

50. LÉVI-STRAUSS, Cl., «Philosophie at anthropologie», Interview de C. Lévi-Strauss, in: Cabiers de Pbilosoplice 1, 1966, p. 55.

51. C'est la thèse de TOULMIN, S., «Reasons and Causes», in: BORGIR, R., CIOFFI, F., Explanation in the Bebatioral Sciences, Cambridge (The University Press), 1970, p. 17 ss. 
y reconnaisse une «force», c'est éluder le fait qu'il est toujours possible, en dépit de la présence de supposées causes, de ne pas agir. Il reste finalement la possibilité de voir dans une règle l'expression d'un besoin de base et de tenir celui-ci pour la véritable cause. Mais s'il en était ainsi, les mêmes causes auraient des effets différents, puisque les « règles 》 des systèmes de parenté sont fort variées. Un tel état de choses est incompatible avec les caractéristiques d'une relation causale.

Une autre façon d'introduire la causalité dans l'analyse structurale est d'attribuer à la structure même une efficacité causale. Une telle causalité n'est plus une causalité mécanique à efficace transitive entre deux événements simples; elle constitue une relation entre le tout (complexe) et ses parties (simples), relation qu'on se propose de définir d'une manière cohérente. Lévi-Strauss refuse explicitement une telle position, mais il postule indirectement l'existence d'un «inconscient structurel » qui est appelé à jouer un rôle semblable. Par contre, les présuppositions que met en jeu une telle «détermination des phénomènes d'une région donnće par la structure de cette région ${ }^{52}$ sont à ses yeux très loin de satisfaire les exigences de l'analyse scientifique. Sans entrer dans les détails de la problématique althusserienne, retenons qu'invoquer la causalité structurale c'est, d'une part, postuler l'existence concrète d'une structure et, d'autre part, concevoir les rapports de cette "réalité » cachée, la cause, à ses manifestations visibles, les effets. Or, en dépit de la signification métathéorique ou formelle qu'on a reconnue au concept de structure, Lévi-Strauss ne semble pas se limiter à ce statut car il confère en outre au concept de «structure»et ce malgré sa propre défense contre Radcliff-Brown - le pouvoir de désigner une réalité qui aurait une existence objective. En cela Lévi-Strauss prépare la route à Althusser, comme l'affirme Geucksmann: "Althusser is the main theorist of structural causality, while Lévi-Strauss demonstrates it pratically ${ }^{53}$. Ceci devra conduire Lévi-Strauss dans une position contradictoire.

Bien sûr, il n'est pas question -- Althusser le souligne à

52. ALTHUSSER, L., BALIBAR, E., Lire le Capital II, op. cit., p. 61.

53. GLUCKSMANN, M., Structuralist Analysis in Contemporary Social Tbougbt, A Comparison of the Theorie of Claude Lévi-Strauss and Louis Althusser, Iondon and Boston (Routledge \& Kegan Paul), 1974, p. 149. 
maintes reprises - de postuler une structure qui existe à part ou à côté des éléments qu'elle détermine, à la manière des idées platoniciennes. En cela Althusser semble également suivre Lévi-Strauss, qui refuse de séparer «forme» (structure) et «contenu». En effet, Lévi-Strauss maintient que la structure est le contenu dans le sens qu'elle représente une propriété (logique) qui est en même temps une propriété du réel ${ }^{54}$. Par contre, pour être déterminante, pour exercer une action, une structure doit jouir d'une certaine autonomie. Elle doit donc être hors de ce qu'elle détermine. Être à la fois dans et hors de ce qu'elle détermine, voilà le statut paradoxal qui caractérise la causalité structurale.

L'interprétation ontologique de la structure, déjà problématique en elle-même, parait être dans le contexte des recherches de Lévi-Strauss une position inconsistante. Pour se dissocier des anthropologues anglais, dont Radcliff-Brown, n'a-t-il pas répété que «quand on parle de structure sociale, on s'attache surtout aux aspects formels des phénomènes sociaux; on sort donc du domaine de la description pour considérer des notions et des catégories qui n'appartiennent pas en propre à l'ethnologie ${ }^{55}$. Et plus loin: «Le principe fondamental est que la notion de structure sociale ne se rapporte pas à la réalité empirique, mais aux modèles construits d'après celle-ci. Ainsi apparaît la différence entre deux notions si voisines qu'on les a souvent confondues, je veux dire celle de structure sociale et celle de relations sociales ${ }^{56}$. La structure est donc avant tout la propriété d'un modèle, c'est-à-dire d'un schéma théorique. Elle ne représente ni une essence ni une substance, ni un événement complexe. S'il en est ainsi, on ne peut guère lui prêter une efficacité causale.

Mais l'usage que fait Lévi-Strauss du concept de modèle n'est pas aussi univoque que dans la citation précédente, ò̀ on lui reconnait soit une fonction descriptive, soit une fonction théorique. En dehors de ce contexte, Lévi-Strauss utilise le terme «modèle» de manière peu orthodoxe, quand il distingue, p.ex.,

54. LÉVI-STRAUSS, C., «La structure et la forme, réflexions sur un ouvrage de Vladimir Propp », in: Cabiers de l'Institut de Sciences Economiques t Appliquées 99, p. 3 ss.

55. LÉVI-STRAUSS, C., Antbropologie structurale, op. cit., p. 304.

56. Ibidem, p. 305. 
des «modèles conscients 》 des «modèles inconscients ${ }^{57}$, entendant par là des « représentations conscientes ou inconscientes 》 des relations ou des structures de parenté. De la sorte, il fait correspondre la structure d'un modèle à une réalité inconsciente. Les régularités qui caractérisent les phénomènes culturels, les régularités que les indigènes ignorent, auraient leurs racines dans leur propre inconscient ${ }^{58}$. Cette activité inconsciente de l'esprit opère fondamentalement de la même manière dans toutes les cultures qui trouvent là leurs contraintes naturelles. Nous avons donc affaire à des facteurs objectifs et non à un principe d'interprétation ${ }^{59}$ du comportement institutionnalisé, car, en tant que facteur, l'inconscient structurel détermine les actions des individus à la manière d'une cause. Mais pour défendre un tel point de vue avec la rigueur scientifique, il aurait fallu expliquer le rapport entre les deux niveaux, celui du comportement conscient et celui de la structure inconsciente. En plus, il aurait fallu justifier ce réductionnisme psychologique qui aboutit en fin de compte à des structures cérébrales, justification difficile pour Lévi-Strauss parce que le réductionnisme en question entre manifestement en conflit avec Les structures élémentaires de la parenté qui est expressément dirigé contre la réduction des phénomènes sociaux à des phénomènes biologiques ou psychologiques. Ceci veut dire qu'on chasse le réductionnisme naturaliste par la grande porte pour le réadmettre par la porte de service. Si Lévi-Strauss veut éviter une telle contradiction ou bien il renonce à l'analyse structurale ou bien il rejette l'existence de la structure sur le mode inconscient et par-delà renonce-t-il à la causalité structurale.

S'il est difficile d'introduire des «causes» au niveau de l'analyse structurale, on peut se demander si le plan des structures constitue une explication ultime ou s'il n'y a pas au-delà des structures d'autres principes qu'il faut invoquer pour répondre à des questions que l'analyse structurale est incapable de traiter, de sorte que le recours à des «causes » devienne nécessaire ${ }^{60}$.

57. Ibidem, p. 308.

58. Ibidem, p. 26.

59. Ibidem, p. 28.

60. Rappelons que Mauss, précurseur de l'analyse structurale et source précieuse de Lévi-Strauss, donnait à son ouvrage Essai sur le don le sous-titre: Forme et raison de l'échange dans les sociétés archaïques, comme s'il 
En examinant, du point de vue épistémologique, Les structures élémentaires de la parenté de Lévi-Strauss on peut, en effet, distinguer dans l'analyse des systèmes de parenté des questions et des problèmes qui appartiennent à deux plans différents: d'une part, il s'agit pour Lévi-Strauss de montrer «comment on peut engendrer toute la variété des structures (élémentaires) connues à partir de quelques composantes simples ${ }^{61}$ et comment on peut, à partir des hypothèses concernant les structures, expliquer l'intégralité des faits problématiques et observés, relatifs à la parenté, notamment l'absence de mariage entre cousins parallèles. D'autre part, Lévi-Strauss s'efforce d'expliquer pourquoi parmi des structures théoriquement possibles certaines seulement sont empiriquement réalisées, et pourquoi certaines sont réalisées de préférence à d'autres ${ }^{62}$. Il faut en plus expliquer l'apparition, sur le fond de structures de "parenté biologique», des structures de parenté par alliance et le changement de ces dernières au cours du temps ${ }^{63}$.

Les derniers problèmes demandent, comme on le verra, une explication qui dépasse le niveau des structures et qui se situe dans un contexte interprétatif, qui jettera une autre lumière sur l'analyse scientifique (structurale). De ce fait, le concept de «structure» sera pris dans un sens spéculatif également; il aura des connotations nouvelles qui seront proprement philosophiques.

Quand Lévi-Strauss s'interroge sur la supériorité, à savoir sur la fréquence plus grande des systèmes à mariage matrilatéral par rapport aux systèmes à mariage patrilatéral, supériorité que l'analyse structurale comme telle n'explique pas, il doit avouer que la prédominance en question «ne semble comporter aucune solution ${ }^{64}$ du point de vue logique, c'est-à-dire si on

voulait indiquer par ces termes, qu'il n'est pas suffisant de décrire des structures de l'échange, mais qu'il faut également rendre raison de l'existence même de ces structures.

61. IADRIÈRE, J., «Le structuralisme entre la science et la philosophie», in : Tijdscbrift voor Filosofie 33, 1971, p. 72. On suit ici pour l'essentiel la distinction que discute Ladrière dans son article.

62." LÉVI-STRAUSS, C., Les structures élémentaires, op. cit., ch. XXVII, XXVIII, XXIX, pp. 505-570.

63. LÉVI-STRAUSS, C., Anthropologie structurale, op. cit., p. 342 ss.

64. LÉVI-STRAUSS, C., Les structures ćlémentaires, op. cit., p. 507. 
se limite au plan des structures. Mais l'étude structurale des deux systèmes de parenté met d'abord en relief leurs différences formelles. Ainsi constate-t-on que le premier présente une «meilleure $»$ structure que le second, car il constitue le développement le plus complet du principe de croisement, de la relation formelle d'alternance des sexes, dont dépend l'existence des cousins croisés, tandis que le second, avec deux paires asymétriques dans un quatuor comprenant alliance et descendance de deux lignées et de deux générations n'est qu'à moitié conforme à l'ordretype ${ }^{65}$. Cette différence formelle-n'est pas vraiment explicative. On doit en plus la comprendre à partir d'un critère ou d'une exigence fondamentale gouvernant l'organisation des structures $^{66}$. Ce critère est, négativement, l'interdit de l'inceste (« renoncer à un lot de femmes immédiatement disponibles»), positivement, la loi de l'alliance ( assigner positivement un cercle de parenté »). La loi de l'alliance n'est pas elle-même de nature structurale, mais elle explique pourquoi les structures s'organisent de telle ou telle manière; elle fournit la condition générale à laquelle doivent se soumettre les structures pour être admissibles. Elle n'exprime plus un schéma d'organisation, mais un canon selon lequel ces schémas peuvent et doivent être formés.

C'est donc par rapport à l'exigence fondamentale de réciprocité, énoncée dans la loi d'alliance, qu'on peut mesurer véritablement la différence de structure et de fonctionnement entre un système matrilatéral et un système patrilatétal et expliquer la prédominance du premier. En effet, lorsque le mariage se fait avec la cousine croisée patrilatérale, ceci représente un échange au comptant puisque la dette est rendue à la génération suivante (voir le schéma de la note 65); par conséquent, le mariage patrilatéral établit une réciprocité entre deux lignées seulement; il instaure un cycle court et stérile du point de vue de l'alliance.

65. Ibidem, pp. 511-12. Les schémas des deux quators sont:
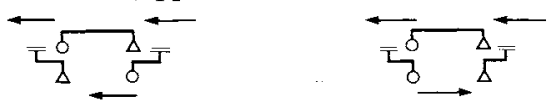

les flèches indiquent la direction de l'échange des femmes.

66. SIMONIS, Y., Claude Lévi-Strauss ou la «Passion de linceste»: Introduction au structuralisme; Paris (Aubier-Montagne), 1968, serait en désaccord avec cette interprétation. Dans son exposé, c'est la structure qui explique la prohibition de l'inceste (p. 55). Mais la suite de l'argument qu'on expose ici établira le contraire. 
Ce mariage offre, par contre, une plus grande sécurité par rapport au mariage matrilatéral qui constitue un risque, car il instaure un cycle long et assure la réciprocité seulement au bout de plusieurs générations après avoir tissé des liens entre plusieurs lignées. Si l'inceste constitue la limite en deça de laquelle il n'y a plus de réciprocité, la loi de l'alliance stipule la maximisation de la réciprocité dont les deux systèmes constituent les deux extrêmes: la structure patrilatérale (cycle court, stérilité, sécurité) représente un minimum, la structure matrilatérale (cycle long, fécondité, risque) recèle des virtualités inépuisables et vise vers un maximum. De ces considérations Ladrière tire la conclusion suivante: « $\mathrm{La}$ dominance du système matrilatéral signifie que, dans la majorité des cas, les groupes humains ont choisi la fécondité, c'est-à-dire l'extension des alliances, au détriment de la sécurité : ils ont préféré l'universalité à la stabilité. La préférence pour le système matrilatéral représente l'affirmation de la loi d'alliance. Celle-ci indique une finalité à laquelle se soumettent les structures ${ }^{67}$.

Homans et Schneider ${ }^{68}$ voient dans le recours à la finalité qui est certainemeni présente chez Lćvi-Strauss, quoiqu'il ait voulu se défendre contre cette interprétation philosophique ${ }^{69}$, une explication téléologique. Elle fournirait les conditions spéciales dans lesquelles les sociétés adoptent l'un ou l'autre des deux systèmes discutés. Ces conditions seraient des caluses finales dans le sens que différentes structures sont adoptées en the d'augmenter la réciprocité et la solidarité organique dans le groupe. Certaines structures serviraient mieux cette finalité et par conséquent elles seraient plus fréquenment réalisćes. Lévi-Strauss affirmerait donc qu'une institution est telle parce qu'elle est «bonne pour la société dans le sens qu'elle crée la solidarité organique, et de ce point de vue certaines institutions sont meilleures que d'autres $\gg{ }^{70}$. Mais suivant la théorie aristotélicienne sur les causes,

67. LADRIÈRE, J., «Le structuralisme », op. cit., p. 74 .

68. HOMANS, G.C., SCHNEIDER, D.M., Marriage, Autbority', and Final Causes, New York, (The Free Press), 1955, republié dans: HOMANS, G.C., Sentiments and Activities, Essays in Social Science, New York (The Free Press), 1962, ch. 14, pp. 202-255, texte qu'on utilisera ici.

69. NEEDHAM, R., Structures and Sentiment, op. cit., p. 27 ss.

70. HOMANS, G.C., Sentiments and activitics, op. cit., p. 213.

71. LÉVI-STRAUSS, C., Les structures élémentaires, op. cit., p. 116. 
« la maison est là en vue de constituer un habitat pour l'homme; mais elle est là aussi parce que les constructeurs ont mis pierre sur pierre ». Il s'ensuit qu'à toute cause finale correspond une cause efficiente qui est nécessaire pour compléter une explication téléologique. Mais chez Lévi-Strauss, il n'est jamais question de causes efficientes; il n'est par exemple jamais question de faire des structures inférieures les causes des structures supérieures. $\mathrm{Ne}$ dit-il pas que l'institution du mariage « résulte d'une série d'accidents historiques pour eux-mêmes dépourvus de signification $\gg^{71}$. Il se propose néanmoins d'expliquer le «passage de l'état de nature qui ne connaît que l'indivision et l'appropriation » à l'état de culture où il y a division et réciprocité ; il est pour lui question de reconstittier « scrupuleusement la séquence» de cette évolution ${ }^{72}$. Une explication diachronique saisirait, espère Lévi-Strauss, l'évolution qui dirige les structures «vers un but ${ }^{i 3}$. Il admet donc une finalité, mais sans causes efficientes que Homans et Schneider veulent introduire en guise de complément.

L'explication par des causes efficientes de Homans et Schneider s'appuie sur le bien connu «complexe patrilinéaire». Ils posent l'hypothèse que tout système matrilatéral est en même temps patrilinéaire. Or, dans une filiation patrilinéaire, le père exerce le droit, l'autorité sur son fils; de là résulte la distance de son fils à son égard et à l'égard de sa parenté. Selon Homans et Schneider, cet état de chose constituerait la motivation adéquate pour que le fils se range du côté de la mère et cherche une partenaire dans ce cercle de parenté, d'où la préférence matrilatérale. La motivation individuelle est par conséquent la cause (efficiente) qui détermine l'adoption d'une structure matrilatérale. Ce n'est pas le lieu ici de reprendre la critique que LéviStrauss a lui-même formulée quant à cette théorie du complexe patrilinéaire (concernant la fausseté de l'hypothèse de départ) ${ }^{i .4}$, ni la réfutation point par point des arguments de Homans et Schneider par Needham qui aboutit à la conclusion que l'explication psychologique de Homans et Schneider n'est pas une explication en termes de causes efficientes et que ce n'est même

72. Ibidem, p. 562.

73. Ibidem, pp. 564-65.

74. LÉVI-STRAUSS, C., Anthropolngie structurale, op. cit., p. 47 ss., 344-/45, note 1 . 
pas une véritable explication. Sans chercher du côté d'une éventuelle réduction à des causes psychologiques, il est possible d'envisager un autre usage de la catégorie de la finalité dont se réclame implicitement Lévi-Strauss. Il reste à voir par la suite si cet autre traitement de la téléonomie des structures de parenté laisse une place pour la causalité.

L'interprétation en question s'obtient en passant par la «théorie » cybernétique telle que Talcott Parsons l'esquisse dans sa théorie générale de l'action ${ }^{75}$. Parsons et les cybernéticiens se représentent la réalité faite d'architectures, de systèmes d'actions ${ }^{76}$ qui se superposent hiérarchiquement suivant leur degré de complexité, allant des systèmes physiques, systèmes organi. ques, systèmes de personnalité, systèmes sociaux jusqu'aux systèmes culturels. Chaque système possède en vertu de ses proprićt'́s structurelles une indépendance relative par rapport aux autres systèmes; chaque niveau possède des mécanismes d'autocontrôle qui maintiennent la structure dans un équilibre oscillant à l'intérieur de limites relatives à un critère qui régit la structure en question. Pour les systèmes de parenté, c'est l'interdit de l'inceste qui dicte ces limites. Les systèmes entretiennent, d'une part, des rapports horizontaux (avec d'autres systèmes du même degré de complexité), d'autre part, des rapports verticaux (avec des systèmes d'un degré inférieur ou supérieur). Ces derniers rapports forment des circuits de rétroaction: une structure d'un niveau donné obtient son support ( «input») des structures d'un niveau inférieur, pourvu que celles-ci possèdent le degré de complexité approprié, et elle exerce en retour un contrôle ( ontput») dirigé vers le système inférieur) sur les structures-support, mais dans les limites qui garantissent l'autonomie structurelle du niveau en question. Ces mécanismes de contrôle affectent la circulation de l'information (femmes, biens, paroles, etc.) à travers les réseaux d'échange.

Du point de vue strictement scientifique, on peut se représenter la nature comme un système total, lui-même fait d'un

75. PARSONS, T., «An Outline of the Social System», op. cit., et «System Analysis: Social Systems», in: SILLS, D.L., (ed.) International Encyclopedia of the Social Sciences, New York, (The Free Press), 1968, vol. 15, pp. $458-473$.

76. Le terme «action» ne connote ici pas seulement des proprićtés anthropologiques; il est pris dans un sens très large incluant des «actions physiques». 
empilement de structures partielles; et rien n'empêcherait de penser qu'une «structure de complexité donnée ne peut se réaliser que sur la base de structures appropriées supposćes déjà réalisćes» et que «le niveau inférieur figure comme condition nécessaire de l'apparition du niveau supérieur ${ }^{7 \tau}$; mais il n’est pas encore une condition suffisante. Si l'explication va ainsi, il faut forcément invoquer l'émergence de proprićtés nouvelles, c'est-à-dire d'une complexité plus grande qui ne s'explique pas entièrement par le conditionnement (nécessaire mais pas suffisant) des structures d'un degré de complexité moindre ${ }^{78}$. Si les rapports entre les niveaux de structures font intervenir des phénomènes d'émergence, celle-ci est irréductible à une cause dans un sens précis, p.ex. au sens de Mackie ou de Kim.

Mais par le biais de l'émergence on peut attribuer aux structures une dimension dynamique. La biérarchie des structures, où les structures de niveau inférieur contribuent à rendre possibles des structures supérieures et contribuent ainsi à l'émergence d'une complexité croissante, peut être comprise comme transformation diachronique d'une structure qui subit un processus métamorphique. On peut s'arrêter au «constat» de la complexité croissante plus on monte dans l'échelle; mais il y a moyen aussi de postuler un processus de complexification ou un processus de finalisation. Pour revenir aux structures de parenté de LéviStrauss, la question serait de savoir si les structures possèdent effectivement une dynamique interne qui les propulse au-delì d'elles-mêmes, et il faut s'interroger dans quelle mesure la loi de l'alliance est un principe explicatif (au sens spćculatif cependant) suffisamment adéquat, propre à rendre compte d'un tel processus sans recourir à la causalité.

Quand Lćvi-Strauss traite de la prédominance du système matrilatéral sur le système patrilatéral, il nous montre que ce dernier, qui constitue un cycle d'échange court, a des propriétés

77. LADRIÈRE, J., «Le structuralisme », op. cit., p. 99.

78. S'il est question ici d'émergence, ce n'est évidemment pas très précis aussi longtemps qu'on n'est pas en mesure de définir l'occurrence d'une proprićté (W), dite «úmergente», relativement à une théorie (T), à une relation partie-tout (Pt) et une classe de propriétés (G) de sorte que (W) n'est pas déductible à laide de $(T)$ et d'une caractérisation de la relation partie-tout eu égard de toutes les proprićtés de (G), cf. HEMPEL, C.G., Aspects of Scientific Explanation, op. cit., pp. 259-64. 
structurelles telles que le «système» ne peut jamais prétendre embrasser la totalité, qu'il engendre de ce fait une multitude de petits systèmes clos qui se juxtaposent, sans jamais réaliser une structure globale ${ }^{79}$. Il montre que la stérilité du système patrilatéral tient au fait que le progrès dans le degré d'intégration est fonction d'un progrès mécanique, c'est-à-dire d'un accroissement numérique du nombre des participants, tandis que le système matrilatéral, qui constitue un cycle long, est fécond parce qu'il peut «augmenter la solidarité tout en gardant la même extension et la même constitution du groupe ${ }^{80}$. En disant cela n'affirme-t-on pas en même temps que toutes les structures partielles ne sont pas également appropriées pour permettre l'émergence d'une structure plus globale de réciprocité, et que certaines constituent un point terminal définitif (comme il y a du reste des lignées évolutives définitivement arrêtées)? L'idée d'émergence ne signifie-t-elle pas évolution qui traverse, suivant la loi de l'alliance, les systèmes de parenté et qui les «projette» vers un but toujours plus loin que l'étape atteinte (pourvu que les conditions nécessaires soient réalisées)? II ne faut pas oublier que le problème majeur de Lévi-Strauss est de rendre compte du passage de la nature à la culture. Sur la base de structures génétiques - la famille biologique -- appropriées émergent des structures d'alliance, et certaines de celles-ci sont aptes à générer des structures de plus en plus vastes ${ }^{81}$.

Dans un tel contexte, la notion d'émergence reste relativement vague. Elle peut sous-entendre qu'il y a des «causes» à l'oeuvre dans les structures qui les conduisent au-delà d'ellesmêmes vers un but quì est lui-même recommencement. De la sorte, la notion d'émergence semble embrasser à la fois l'idée d'une cause efficiente comme celle d'une cause finale. Mais il faut bien remarquer que nous nous trouvons maintenant sur le terrain de la spéculation où la causalité peut éventuellement retrouver la place qu'elle occupait avant d'être exportée dans les sciences empiriques. Et dans ce cas qu'est-ce qui nous empêcherait de postuler que ce qui émerge dans ce processus de finalisation est ce qui appartient proprement à l'homme et à la

79. LÉVI-STRAUSS, C.,Les structures élémentaires, op. cit., p. 512.

80. Ibidem, p. 509.

81. Ibidem, pp.564-65. 
culture, à savoir la liberté, cause par excellence puisqu'elle ne s'insère pas dans une série déjà commencée, mais qu'elle est, en tant que spontanéité, «une puissance de commencer absolument un état et par suite aussi une série de conséquences de cet état ${ }^{82}$.

Université du Québec à Trois-Rivières.

82. KANT, I., Kritik der reinen Vernunft, Hamburg, Verlag (Félix Meiner) 1956, p. 462. 